\title{
DESIDRATAÇÃO E BALANÇO HÍDRICO EM MEIA MARATONA
}

\author{
GRAD. HERIKSON ARAUJO COSTA
}

Curso de Educação Física, Centro de Ciências da Saúde, Universidade Federal do Maranhão (São Luís - Maranhão - Brasil)

E-mail: heriksoncosta@gmail.com

\section{EDNEI COSTA MAIA}

Curso de Medicina, Centro de Ciências da Saúde,

Universidade Federal do Maranhão

(São Luís - Maranhão - Brasil)

E-mail: ednei.maia@hotmail.com

\section{RAPHAEL FURTADO MARQUES}

Curso de Educação Física, Centro de Ciências da Saúde,

Universidade Federal do Maranhão (São Luís - Maranhão - Brasil)

E-mail: marques.raphaf@gmail.com

\section{AYLA FLÁVIA GUIMARÃES FRAZÃO}

Curso de Educação Física, Centro de Ciências da Saúde,

Universidade Federal do Maranhão (São Luís - Maranhão - Brasil)

E-mail: aylaflavia@hotmail.com

\section{ESP. JUREMA GONÇALVES LOPES DE CASTRO FILHA}

Especialização em Ciências e Medicina do Esporte,

Centro de Ciências da Saúde, Universidade Federal do Maranhão

(São Luís - Maranhão - Brasil)

E-mail: jurema_filha@hotmail.com

\section{DR. FRANCISCO NAVARRO}

Departamento de Educação Física, Centro de Ciências da Saúde, Universidade Federal do Maranhão

(São Luís - Maranhão - Brasil)

E-mail:francisconavarro@uol.com.br

\section{DR. MARIO NORBERTO SEVILIO DE OLIVEIRA JUNIOR}

Departamento de Educação Física, Centro de Ciências da Saúde,

Universidade Federal do Maranhão

(São Luís - Maranhão - Brasil)

E-mail:msevilio@hotmail.com 


\section{RESUMO}

O objetivo deste estudo foi avaliar o nível de desidratação a partir do balanço hídrico e da Densidade da Urina $\left(D_{u}\right)$ em corredores de meia maratona. Participaram do estudo 18 indivíduos do sexo masculino. A ingesta hídrica foi "ad libitum" e realizou-se análise da $D_{u}$ no início e ao final da corrida, bem como medidas de pesagem e estatura. As condições ambientais (33,30 $\pm 5,30^{\circ} \mathrm{C}$ e 57,50 $\pm 13,60 \%$ de URA) foram registradas por meio de termo-higrômetro digital. Os indivíduos terminaram e começaram a prova desidratados, havendo uma diferença significativa no peso corporal (4, I 1\%). Conclui-se que a ingesta hídrica "ad libitum" não foi suficiente para a manutenção do balanço hídrico.

PALAVRAS-CHAVE: Desidratação; Densidade da urina; Meia maratona; Balanço hídrico.

\section{INTRODUÇÃO}

Em torno de 25\%, no máximo, da energia química oriunda da oxidação dos nutrientes durante a caminhada rápida ou a corrida se transformam em energia mecânica, responsável pelo movimento, sendo o restante transformado imediatamente em energia térmica. Contudo, inclusive a energia mecânica é também transformada em energia térmica posteriormente. Portanto, 100\% da energia é transformada em calor (CARVALHO; MARA, 2010 , p. |44- |48). A transferência de calor corporal ao meio externo pode ocorrer através de quatro mecanismos básicos: radiação, condução, convecção e evaporação. A evaporação representa a principal defesa contra o superaquecimento, pois é responsável por aproximadamente $80 \%$ da perda total de calor quando se está fisicamente ativo. (WILMORE; COSTILL $200 \mathrm{I}$ ).

Ao longo de uma hora de exercício extenuante em ambiente quente, ocorre perda significativa de água através do suor, mesmo ao se considerar o exercício realizado em ambiente menos ameaçador do ponto de vista térmico, como por exemplo, a natação, pode ser da ordem de 0,5-I,5 litros. (MACARDLE; KACTH; KATCH, 20 I I). Segundo Wilmore e Costill (200 I ) uma elevada taxa de transpiração reduz o volume sanguíneo, o que limita o volume de sangue disponível para suprir as necessidades dos músculos e para impedir a elevação da temperatura corporal, contribuindo assim para a potencial redução de desempenho. A desidratação severa, por sua vez, pode limitar a transpiração subsequente e tornar o indivíduo suscetível aos problemas de saúde relacionados ao calor.

Os distúrbios do equilíbrio hidroeletrolítico podem afetar a capacidade de tolerar o exercício prolongado. A perda de água através do suor induzida pelo exercício pode levar à desidratação dos compartimentos intracelular e extracelular. Mesmo desidratação leve pode aumentar o esforço cardiovascular, traduzindo-se através de um aumento desproporcional da frequência cardíaca no exercício, além de limitar a capacidade corporal de transferir calor dos músculos em contração 
para a superfície da pele, onde pode ser dissipado para o ambiente. Portanto, um deficit hídrico pode diminuir o desempenho, além de aumentar a possibilidade de ocorrer uma complicação térmica(ACSM, 1999). É importante reconhecer os elementos que interferem na qualidade da hidratação, uma vez que a desidratação decorrente do exercício pode ocorrer não apenas devido à sudorese intensa, mas, também, devido à ingestão insuficiente e/ou deficiente absorção de líquidos (HERNANDEZ et al, 2009).

O estado hídrico dos indivíduos deve ser sempre fator a ser considerado quando da realização de eventos esportivos ao ar livre, dada a maior exposição dos participantes aos fatores ambientais. Considerando o risco inerente à progressiva diminuição da água nos compartimentos corporais, o presente estudo tem por objetivo avaliar o estado de hidratação de indivíduos antes e após uma prova de meia-maratona $(21$, I km) mediante determinação das variáveis: massa corporal, gravidade específica e coloração da urina.

\section{MATERIAIS E MÉTODOS}

O presente trabalho se apresenta como um estudo descritivo, de abordagem quantitativa.

\section{SUJEITOS}

Havia 127 corredores do gênero masculino inscritos na meia maratona, entretanto participaram inicialmente da pesquisa aqueles que aderiram à mesma sob a forma de um termo de consentimento livre e esclarecido (TCLE). Dos 127 corredores, somente 45 submeteram-se à pesquisa.

Ao aderir ao estudo, os participantes tinham que cumprir com os critérios de inclusão e exclusão para que pudessem compor a amostra deste estudo. Os critérios de inclusão eram: (I) não fazer uso de substâncias diuréticas e (2) não ter ingerido bebida alcoólica nas 48 horas que antecediam a corrida. Os critérios de exclusão eram: ( I completar toda a prova, (2) refazer os procedimentos de avaliação da desidratação e (3) conseguir informar a quantidade de copos com água ingerida durante a prova.

Com base nas disposições supracitadas, a amostra foi selecionada de maneira não probabilística por conveniência, constituída por 18 indivíduos do gênero mas-

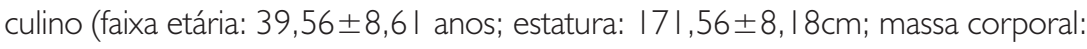
$68,76 \pm 9,65 \mathrm{~kg}$; índice de massa corporal (IMC): 23,26 $\left.2,32 \mathrm{~kg} / \mathrm{m}^{2}\right)$, sendo que todos os indivíduos percorreram os 21 , I km referentes à prova de meia-maratona. 


\section{PROCEDIMENTOS E INSTRUMENTOS}

O estudo foi realizado mediante aprovação pelo Comitê de Ética da Universidade Federal do Maranhão (UFMA), de acordo com as normas da Resolução 196/96 do Conselho Nacional de Saúde sobre pesquisa envolvendo seres humanos. Os participantes foram previamente informados acerca dos objetivos e metodologia da pesquisa por meio de um TCLE. Todos os indivíduos assinaram o referido termo de forma a garantir a participação voluntária no estudo, além de preencher ficha de identificação com dados pessoais.

A caracterização da amostra se deu momentos antes ao início da corrida, tendo como varáveis registradas a idade, estatura e massa corporal dos sujeitos que compunham a amostra. Após os procedimentos de caracterização da amostra, realizou-se a pesagem e coleta de amostra urinária momentos antes do início da corrida. Para tal, os indivíduos eram orientados a realizar o completo esvaziamento da bexiga, coletando então a amostra de urina, para que somente então fosse realizada a aferição da massa corporal. $\bigcirc$ registro da massa corporal foi feito com os sujeitos em posição ortostática, vestidos somente em seu "short" para corrida, com os pés descalços.

Foi utilizada uma balança com estadiômetro digital (WISO, W72I) para aferição da massa corporal e mensuração da estatura. As amostras de urina foram coletadas em copos descartáveis e analisadas em refratômetro (INSTRUTHERM, RTP-20ATC) - 50 $\mu \mathrm{L}$ - para determinar a gravidade específica, este parâmetro se baseou nos pontos de corte estabelecidos pela National Athletic Trainer's associoation (NATA, 2000), (TABELA I). Para o manuseio da urina, foram utilizadas pipetas de 3 $\mathrm{mL}$ descartáveis a fim de transportar a urina até o refratômetro. A higienização do refratômetro foi feita com água destilada e algodão.

Tabela I - Índices de estado de hidratação

\begin{tabular}{l|l|l}
\hline Estado de hidratação & \% peso corporal & Densidade da urina (SG) \\
\hline Eu - hidratação & +1 a -1 & $<1010$ \\
Desidratação mínima & -1 a -3 & $1010-1020$ \\
Desidratação significativa & $-3 a-5$ & $1021-1030$ \\
Desidratação grave & $>-5$ & $>1030$ \\
\hline
\end{tabular}

(NATA, 2000)

Ao término da prova, os indivíduos dirigiam-se novamente ao posto de coleta para realização de nova amostragem de urina e mensuração da massa corporal, informando neste momento a quantidade de água ingerida durante a prova. 
Os sujeitos foram orientados a contar quantos copos d'água ingeriram durante a corrida, como também ingerir todo o volume d’água disponível em cada copo; assim, nós conseguimos quantificar quanto de água foi ingerido, pois os copos tinham capacidade volumétrica de $200 \mathrm{ml}$. As condições ambientais (temperatura e umidade relativa do ar - URA) foram registradas por meio de termo-higrômetro digital (INSTRUTHERM, HT-260). O controle foi iniciado cerca de 30 minutos antes da corrida e encerrado ao término da mesma, sendo o registro da temperatura e URA ambientes efetuado a cada 5 minutos.

Os resultados obtidos neste estudo foram abordados na forma de média (MD) e desvio padrão (DP). A avaliação da diferença na densidade da urina se deu através do teste t de Student para dados pareados, considerando-se estatisticamente significante $p<0,05$.

\section{RESULTADOS}

A caracterização da amostra demonstra que os participantes do estudo formam um grupo homogêneo em relação à idade, peso corporal, estatura e IMC. A média de idade dos indivíduos avaliados foi superior a 39 anos, caracterizando-os como um grupo adulto maduro. A análise do IMC permite classificar os participantes como dentro da faixa de normalidade para indivíduos adultos (JAMES, et al , 200 I)

A temperatura ambiente (TA) e URA registradas no local foram, respectivamente, 33,30 \pm 5,30 ( $\left.{ }^{\circ} \mathrm{C}\right)$ e 57,50 \pm 13,60 (\%). Tais valores caracterizam o ambiente como quente e inapropriado para a prática de exercícios físicos.

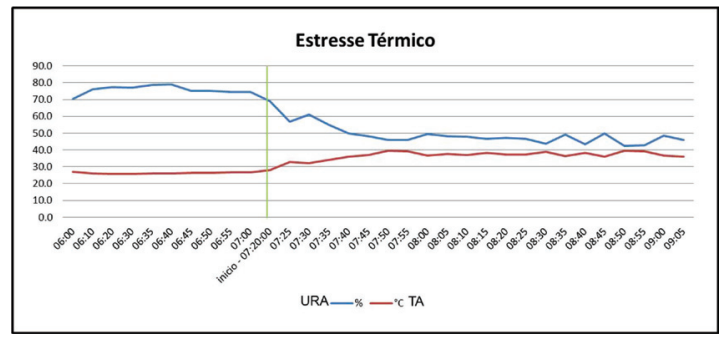

Figura I - Temperatura ambiente (TA) e Umidade Relativa do $\operatorname{Ar}$ (URA) registrada ao longo da corrida. A linha vertical representa o início da corrida.

A análise da desidratação pela densidade urinária (Du) nos mostra que os indivíduos já iniciaram a corrida em estado de desidratação mínima ( $1010 \leq \mathrm{Du} \leq 1020$ ). Não houve diferença estatisticamente significante $(p>0,05)$ entre a Du antes (Du Inicial:

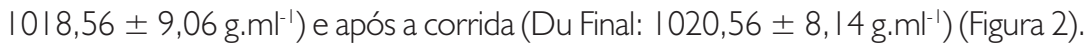




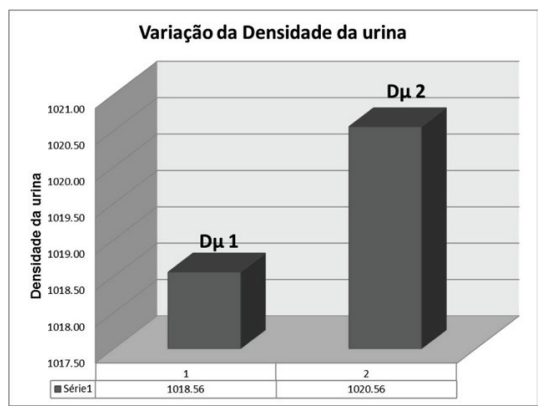

Figura 2 - Variação da Densidade da Urina comparando os valores obtidos antes e após da meia maratona.

Houve diferença estatisticamente significativa $(p \leq 0,05)$ entre o peso corporal inicial e o final, correspondente a uma variação do peso corporal de 4, I I \%, o que é considerado um estado de desidratação significativa (-3 a -5\%). Os indivíduos demoraram cerca de 98 minutos para concluir os 21 , I quilômetros de prova, proporcionado uma taxa de sudorese baixa, quando comparada aos valores encontrados em provas de meia maratona. A reposição hídrica foi 32,92\% acima das recomendações do ACSM, ainda assim, suficiente apenas para repor 2,42\% do líquido perdido durante a corrida (Tabela 2 e Figura 3).

Tabela 2: variação de peso corporal e balanço hídrico - $(\mathrm{n}=18)$

\begin{tabular}{l|l|l|l|l|l}
\hline \multicolumn{3}{c|}{ PESO (KG) } & \multicolumn{2}{c}{ BALANÇO HÍDRICO } \\
\hline \multirow{4}{*}{ MD } & Peso I & Peso F & $\%$ Pp & Liq. Ing. $(\mathrm{ml})$ & TS (L/h) \\
DP & 68,76 & $65,91 *$ & 4,11 & 1716,67 & 0,80 \\
\hline
\end{tabular}

Média (MD), desvio padrão (DP) dos resultados peso inicial - peso I; peso final - peso F; percentual de peso perdido - \%Pp.; líquido ingerido durante a corrida - Liq. Ing. em mililitros - ml; taxa de sudorese - TS em litros por hora - L/h e diferença estatística significativa - * $(P<0,05)$.

\section{DISCUSSÃO}

O objetivo deste trabalho foi avaliar o estado de hidratação de corredores antes e após uma prova de meia maratona. Nossa hipótese de que os corredores já começariam a corrida desidratados, e que o controle de ingestão de líquido pelo mecanismo da sede durante a prova não seria eficiente para restabelecer o balanço hídrico foi confirmada.

O mecanismo da sede tem sido o principal meio de controle de reposição hídrica do ser humano, mesmo já havendo recomendações para esta conduta 
(MARINS, 20 I I). Murray (2007), Sawka e Noakes (2007) colocam que a sede está diretamente relacionada com uma perda hídrica de $2 \%$ do peso corporal. Há relatos na literatura, Costa et al. (20 I I), que mostram que corredores já começam suas provas desidratados, provavelmente isso é devido ao horário em que se dá a maioria das provas de corrida, ao amanhecer do dia, uma vez que durante o sono dificilmente alguém se hidrata.

Para indivíduos que praticam exercícios físicos, é recomendada uma reposição líquida de $600 \mathrm{ml}$ duas horas antes da atividade, acreditando que esta medida seja suficiente para normalizar o nível de hidratação (CASA et al., 2007; ACSM, 2007; MARINS, 201 I).

Os sujeitos desta pesquisa podem ter começado a prova fora de seus pesos reais, pois estavam desidratados. Contudo, pôde-se observar reduções no peso corporal ao final da corrida, apontando para um possível aumento do nível de desidratação. Provavelmente o mecanismo da sede antes e durante o exercício possa não ter sido eficiente para o controle do balanço hídrico. Este desfecho corrobora com os achados de Pitts, Johnson e Consolazio (1944), que comprovaram em seus trabalhos que a sede não é suficiente para repor as perdas hídricas pela sudorese, acarretando em desidratação involuntária. Estes dados podem ser reafirmados pela literatura vigente (MURRAY, 2007; SAWKA; NOAKES, 2007).

O ACSM (2007) sugere uma reposição hídrica de 200 a $250 \mathrm{ml}$ de água a cada 15 ou 20 minutos de exercício físico. Para Marins (20l I ), mesmo sendo obedecidas essas recomendações, é muito improvável que se consiga manter constante a homeostase hídrica, isto porque a capacidade de absorção de líquidos dificilmente é superior a $\mathrm{IL} / \mathrm{h}$, enquanto que a taxa de sudorese facilmente atinge $2 \mathrm{~L} / \mathrm{h}$. Este autor reforça que as atividades que proporcionam um impacto com o solo, como basquetebol e corrida, dificultam ainda mais o esvaziamento gástrico, principalmente em indivíduos sedentários, devido a um refluxo de forças, de forma a manter todo o trato gastrointestinal em constante movimento ondulatório em cada passada.

As afirmações de Marins (20 I I) supracitadas corroboram com os resultados encontrados no estudo de Pereira (20 I I), no qual encontrou uma taxa de sudorese equivalente a I,44L.h $\mathrm{h}^{-1}$ ou 2,44 litros de suor durante os I I I,9 minutos de corrida e uma reposição hídrica de apenas 800 mililitros, estando $28 \%$ abaixo das recomendações. Entretanto, esses resultados não se reproduziram em nossa pesquisa, em que se pode observar uma perda média equivalente a I,07 litros de suor, em contrapartida, a reposição hídrica foi igual a I,77 litros, sendo, portanto, superior a taxa de sudorese. Possivelmente, isto pode ter ocorrido devido ao deficit hídrico encontrado nos sujeitos antes da prova, bem como por um aumento deste deficit durante a corrida. De acordo com Armstrong et al. (2000), a taxa de sudorese 
diminui quando há uma redução no volume de líquido intracorporal equivalente a $1 \%$ do peso corporal.

Nossos resultados apontam uma redução de $4,11 \%$ de peso corporal, o que caracteriza um quadro de desidratação significativo. $\bigcirc$ mecanismo da sede disparado por esta desidratação foi responsável por uma reposição hídrica 35\% acima das recomendações oficiais do ACSM (2007). Entretanto, essa reposição não foi suficiente para restabelecer a homeostase hídrica, ou seja, deixar os indivíduos eu-hidratados ou normo-hidratados.

Os resultados da desidratação, aqui encontrados, pelo método da Du, apontam níveis de desidratação mínima antes e após a corrida, não havendo diferenças significativas entre os momentos citados. Contudo, a desidratação avaliada pelo método da variação de peso corporal mostrou diferenças significativas, com os sujeitos saindo de uma desidratação mínima para uma desidratação significativa.

O fato da Du não ter variado significativamente durante a corrida, provavelmente pode ser atribuído à redução na taxa de sudorese, que por sua vez diminui a variação na Du. Isto ocorre devido a uma diminuição do fluxo sanguíneo aos rins, acarretando em diminuição da taxa de filtração glomerular que leva a uma manutenção da Du (GUYTON; HALL, 20I I). Provavelmente, a diferença encontrada nos resultados das avaliações da desidratação a partir da variação de peso corporal e Du podem ser atribuídas às diferenças das técnicas entre os dois métodos.

A desidratação pode ser um dos fatores limitantes do desempenho físico (MCARDLE; KATCH; KATCH, 20I I). Silva et al. (20l0), analisando o efeito da desidratação sobre o desempenho físico, verificaram fortes correlações negativas entre essas duas variáveis, ou seja, quanto maior era o nível de desidratação, menor seria o desempenho durante o exercício físico. A desidratação associada ao estresse térmico pode ser potencializada, nesse caso, o estresse térmico aparece como um agravante à queda de desempenho durante um exercício físico.

O estresse térmico encontrado durante a prova não nos permite caracterizar esse ambiente como termoneutro, pois apesar de a média de URA ter sido superior a $50 \%$, a temperatura registrada se mostrou demasiado elevada $\left(33,30^{\circ} \mathrm{C}\right)$, o que oferece um risco e um desafio maior aos corredores. A termorregulação humana também é possível nestes ambientes, o que permite a realização de exercícios físicos durante maior tempo (ARMSTRONG, 2000).

Foram registrados no decorrer da prova picos extremos de temperatura $\left(38^{\circ} \mathrm{C}\right.$ ), assim como momentos de URA abaixo de 50\%. A combinação desses extremos coloca os indivíduos frente a um ambiente bastante desafiador para prática de atividade e exercício físico (MACIEIRA, 2009).

Mecanismos fisiológicos e comportamentais estão por trás da queda de desempenho durante exercícios físicos realizados em situações estressoras, como é o 
caso da desidratação e estresse térmico. Morante e Brotherhood (2008) mostraram em jogadores de tênis sob uma temperatura ambiental de $38^{\circ} \mathrm{C}$, uma diminuição do trabalho físico, mecanismo comportamental, e redução da taxa de sudorese, mecanismo fisiológico, com um significativo aumento da temperatura corporal. Contudo, eles não monitoraram a URA, variável esta responsável pela evaporação do suor e consequentemente resfriamento da pele. Sigurbjorn et al. (2003) mostraram que uma variação de 25 a $40^{\circ} \mathrm{C}$ não é capaz por si só de aumentar a temperatura corporal central, sendo necessário uma combinação com estresse físico.

Os mecanismos de termorregulação e a aptidão física sofrem influência da idade cronológica, uma vez que os níveis de aptidão decrescem (OLIVEIRA; ANJOS, 2008) e o limiar de sudorese aumenta com o aumento da idade (ARMSTRONG, 2000). Em nosso estudo os sujeitos se caracterizaram como adultos maduros, isso implica em uma provável redução da taxa de sudorese, limitando assim, o desempenho dos corredores. Contudo, a relação entre as variáveis desempenho, idade e os mecanismos de termorregulação não foi objeto de estudo deste trabalho.

\section{CONCLUSÃO}

A partir dos dados apresentados, conclui-se que o mecanismo da sede não foi eficiente para restabelecer o balanço hídrico dos sujeitos antes e durante a corrida, uma vez que os sujeitos já começaram a prova desidratados, e que a desidratação associada ao estresse térmico pode determinar uma redução da taxa de sudorese.

\section{Dehydration and Water Balance in Half Marathon}

ABSTRACT: The aim of this study was to evaluate the degree of dehydration from the hydric balance and density of urine (Du) in half marathon runners. The study included 18 male subjects. The fluid intake was "ad libitum" and was held in Du analysis at the beginning and at the end of the race, as well as measurements of weight and height. Environmental conditions $\left(33,30 \pm 5,30^{\circ} \mathrm{C}\right.$ and $57,50 \pm 13,60 \%$ of URA) were recorded by digital hygrometer. The subjects began and finished the study dehydrated, and showed a significant difference in body weight (4, I 1\%). We conclude that the fluid intake "ad libitum" was not sufficient to maintain hydric balance.

KEYWORDS: Dehydration; Density of Urine; Half Marathon; Water Balance.

\section{Deshidratación y el balance hídrico em media maratón}

RESUMEN: El objetivo del estudio fue evaluar el nivel de deshidratación por el balance hídrico y la densidad de la orina (Du) en corredores de media maratón. El incluyó I 8 sujetos masculinos. La ingesta de líquidos fue "ad libitum" y se realizó análisis de Du al antes y al 
después de la carrera, así como mediciones de peso y altura. Las condiciones ambientales $\left(33,30 \pm 5,30^{\circ} \mathrm{C}\right.$ y $57,50 \pm 13,60 \%$ de HR) fueron registradas por termohigrómetro digital. Los individuos comenzaron y terminaron la carrera deshidratados, con una diferencia significativa en el peso corporal (4, I 1\%). Se concluye que la ingesta de líquidos "ad libitum" no era suficiente para mantener el equilibrio hídrico.

PALABRAS CLAVE: Deshidratación; densidad de la orina; media maratón; equilibrio hídrico.

\section{REFERÊNCIAS}

AMERICAN COLLEGE OF SPORTS MEDICINE (ACSM) - Posicionamento Oficial: Distúrbios causados pelo frio e pelo calor durante corrida de longa distancia. Revista Brasileira de Medicina do Esporte, São Paulo, v. 5, n. 03, p. 108-119, mai./jun. 1999.

AMERICAN COLLEGE OF SPORTS MEDICINE (ACSM) - Position Stand: Exercise and fluid replacement. Medicine and Science in Sports and Exercise, Indianapolis, v. 39, n. 2, p. 377-390, Feb 2007.

ARMSTRONG, LE. Performance in extreme enviorments. Champaign: Human Kinetics, 2000.

CARVALHO, T.; MARA, L. S. Hidratação e nutrição no esporte. Revista Brasileira de Medicina do Esporte, São Paulo, v. 16, n. 2, p. |44-|48, mar./abr. 2010.

CASA, D. J et al. Validity of that assess body temperature during outdoor Exercise in the heat. Journal of Athletic Training, v. 42, n. 03, p. 333-342, 2007.

COSTA, H. A. et al. Nível de desidratação a partir do balanço hídrico e gravidade específica da urina em corredores de rua em ambiente quente. In: 34 SIMPÓSIO INTERNACIONAL DE CIÊNCIAS DO ESPORTE, 20 I I, São Paulo. Especulações e evidências em atividade física e esporte, 2011.v. 19. p. 197-197.

GUYTON, A. C.; HALL, J. E. Tratado de fisiologia médica. 12. ed. Rio de Janeiro: Elsevier, 20I I HERNANDEZ, A. J et al. Modificações dietéticas, reposição hídrica, suplementos alimentares e drogas: comprovação de ação ergogênica e potenciais riscos para a saúde. Revista brasileira de medicina do Esporte, São Paulo, v. 15, n. 03, p. I- 12, mar./abr. 2009.

JAMES, P. T. et al. The worldwide obesity epidemic. Obesity research, New York, v. 9, n. 4, p. 228-233, nov. 2001

MACARDLE, D. M; KACTH, F. I; KATCH, V. L. Nutrição para o desporto e o exercício. Rio de Janeiro, Guanabara, 20। I.

MACIEIRA, J. Calor, desidratação e degradação Muscular no Exercício. Revista Portuguesa de Fisioterapia No Desporto, São Domingos de Rana, v. 03, n. 02, p. 22-31, jan./jun. 2009.

MARINS, J. C. B. Hidratação na atividade física e no esporte: equilíbrio hidromineral. São Paulo: Editora Fontoura, 201 I. 
MORANTE, S. M; BROTHERHOOD, J. R.: Autonomic and behavioural thermoregulation in tennis. Brazilian Journal of Sports Medicine. v. 42, n. 02, p. 679-785, may. 2008.

MURRAY, B. Hydration and physical performance. Journal of the American College of Nutrition, Clearwater, v. 26, n. 05, p. 542-548, Oct, 2007.

NATIONAL ATHLETIC TRAINER'S ASSOCIATION (NATA) - Position Statement: Fluid replacement for athletes. Journal of athletic training, Dallas, v. 35, n. 02, p. 212-24, jul./set. 2000.

OLIVEIRA, E. A. M; ANJOS, L. A. Medidas antropométricas segundo aptidão cardiorrespiratória em militares da ativa, Brasil. Revista Saúde Pública, São Paulo, v. 42, p. 21 7-223, abr. 2008.

PEREIRA, E. R. Avaliação do estado de hidratação de corredores amadores antes e após uma prova de meia-maratona. 201 I. 58 f. Dissertação (Mestrado em Ciência do esporte) - Universidade Federal de Minas Gerais, Belo Horizonte, 201 l.

PITTS, G. C.; JOHNSON, R. E., CONSOLAZIO, F. C. Work in the heat is affected by intake of water salt and glucose. American Journal of Physiology, California, v. 142, n. 02, p. 253-259, set. 1944.

SAWKA, M. N., NOAKES, T. D. Does dehydration impair exercise performance? Medicine and Science in Sports Exercise, Indianapolis, v. 39, n. 8, p. 1209-1217, Aug, 2007.

SIGURBJORN, A. et al. Relation of heart rate to percent VO2 peak during submaximal exercise in the heat. Journal of Applied Physiology, Bethesda, v.94, n. 03, p. I I62-I I 68, mar. 2003.

SILVA, A. I. et al. Nível de desidratação e desempenho físico do árbitro de futebol no Paraná e São Paulo. Revista Brasileira de Fisiologia do Exercício, Ivaiporã, v. 9, n. 3, p. | 48- I 55, Jul/ Set, 2010.

WILMORE, J. H.; COSTILL, D. L. Fisiologia do esporte e do exercício. São Paulo: Manole, 200 I.

Recebido em: 26 dez. 2012

Aprovado em: 5 set. 2013

Endereço para correspondência:

Herikson Araujo Costa

Rua $1^{\circ}$ de maio, No $17 \mathrm{~A}$

Bairro Vila Embratel

São Luís - MA

CEP: $65080-140$ 
